\title{
Modern Physics As A New Step In Dialogue Of Man With Nature
}

\author{
V. K. Voronov \\ Irkutsk State Research Technical University \\ Irkutsk, Russia.
}

\begin{abstract}
The paper describes the content of a set of textbooks entitled "Modern Physics" consisting of three monographs, which cover the main advancements of physics approximately for last fifty - sixty years (since the middle of the twentieth century). The first textbook contains ten chapters. The second book includes five chapters. In the third book, consisting of three parts, physical foundations of nanotechnologies are discussed. The textbooks are written on the basis of the material, which is selected from various sources (reviews, monographs). The authors mainly discuss the papers published in "Advancements of physical sciences" journal. The total volume of three books is about eighty printed pages. Justification of the significance of the work is also provided. In this line, it is noted that rapid growth of scientific information and everstrengthening integration of various branches of science lead to the problems of methodological character, which solution is connected with publication of course books. It is also underlined that since the middle of the twentieth century, a number of new branches of physics, including the nonlinear optics, physics of open systems, quantum information, molecular structure and dynamics, has been developed. There is rather extensive scientific literature related to branches of physics. These are mainly original papers and reviews published in specialized journals. However, as a rule, this literature is intended for experts. As for training materials, they are almost absent.
\end{abstract}

Keywords: modern physics; condensed state; nanomaterials.

\section{INTRODUCTION}

A crucial point in the development of modern science is the ever-increasing amount of information. Having arisen in the ancient world due to the requirements of public practice, the science has turned into the productive force and the major social institute exerting significant influence on all spheres of society and culture as a whole. Since seventeenth century, the volume of scientific information doubles approximately everyone ten - fifteen years (growth of discoveries, number of researchers, etc.). Tremendous growth of scientific information leads to more and more increasing gap between the level of knowledge reached by science and the level training in universities. In one of his presentations, Professor S.P. Kapitsa suggested that each generation should write its own textbook on physics. This brings up the questions: "Has time for writing such textbook come and, if yes, is the modern generation (living in the end of the twentieth - the beginnings of the twenty first century) ready to do it? And the most important what is the content of such textbook?"

Speaking about history of physics, the famous American physicist and science expositor Jay Orear has stated that one can distinguish (quite randomly) three periods in this history: classical, new and modern [1]. By the end of the 19th century, such sections of physics as mechanics, thermodynamics, electromagnetism, optics and hydrodynamics have been in detail studied. In general, it seemed that the theoretical understanding of these sections was quite complete and one could hardly expect here any new important discoveries. These sections were commonly referred to as classical physics. 
At the very end of the nineteenth century and during first three decades of the twentieth century a number of astonishing discoveries has been made in physics. For instance, the radioactivity has been revealed. Further, this phenomenon was used for the studies of atomic structure. Development of the relativity theory has allowed the old ideas about space and time to be revisited. Attempts to describe a structure of atoms have led to the creation of the quantum theory. This period, during which all character of physical researches has changed, is called the period of new physics.

In the thirties of the twentieth century the radio emission of stars was observed for the first time observed. Also, the neutron and nuclear fission were discovered. These and other discoveries have led to accumulation of a huge number of data in the new fields of physics, and this trend keeps preserving nowadays. Similar development of physical researches, which is accompanied by further discoveries and generation of new ideas, has resulted in modern physics.

Another distinctive feature of modern natural sciences (along with the growth of information), is the ever-increasing integration of scientific research. Such a trend makes the division of natural sciences into strictly specific branches even more and more conditional. Nevertheless, the physics, studying the simplest and most general properties of a material world, continues to play a key role in natural science. At the same time, other branches of natural science keep preserving their specifics. The above two features (rapid growth of scientific information and ever-increasing integration of various sections of natural sciences) put forward methodological problems, which could be solved by publishing educational materials. The more important fact is that the emphasis very often is placed on "how to teach". However, the real problem is another: "To what to teach?"

All the above stated has prompted the author of this work together his colleagues, Professors A.V. Podoplelov and R.Z. Sagdeev, to write a textbook, which would cover the achievements of modern physics approximately for fifty - sixty years (beginning from the middle of the twentieth century). This period is characterized by appearance of a number of new branches of knowledge, in particular, nonlinear optics, physics of open systems, quantum information, and branches related to the studying of molecular structure and dynamics. There is rather vast scientific literature related to different aspects of physics (original papers and reviews published in specialized journals). However it is mainly intended for experts. As for the study materials devoted to the abovementioned branch of knowledge, it is almost lacking.

The abovementioned books are based of three textbooks [2-4] recommended for publication by scientific and methodical council for physics of the Ministry of Education and Science of the Russian Federation. They are written basing on the materials, which have been selected from various sources, mainly from the reviews published in the "Advances in Physical Sciences" journal. The list of references is given in the end of each chapter. In some cases, the list of references contains publications, which are discussed briefly or not discussed at all in the book. But we believe that these references could be useful for deep understanding of the material. Thus, the reader will be able to use a vast list of references devoted to different problems of modern physics.

\section{CONTENT OF «MODERN PHYSICS» TEXTBOOKS.}

The first textbook consists of ten chapters. Chapter 1 deals with the fundamentals of new nonlinear optical effects, which are induced due to intensity of light. There are two reasons causing different results of the interaction between substance and light of low or high intensity. First, in the case of high intensity, multiphoton processes involving absorption of 
several photos in an elementary act, play the major role. Second, high intensities induce the self-action effects changing the initial properties of substance under the action of the propagating light. Chapter 2 is devoted to the basic concepts of the new interdisciplinary research direction, physics of open systems, which was originated owing to the works of outstanding scientists of the nineteenth century. Among them are physicist Ludwig Boltzmann, mathematicians Henri Poincare and Alexander Lyapunov, the biologist Charles Darwin. Chapter 3 of the textbook describes the physics of solids, which holds a special place, since it allows obtaining the data on a structure of matter of living and non-living nature as well as on the materials used in the engineering. In chapter 4 of the book, novel possibilities of wide and multi-faceted applications of semiconductors in various areas of science and engineering are discussed. Chapter 5 is dedicated to a series of new objects of atomic physics attracting everincreasing research attention due to a variety of the physical properties and the phenomena inherent in these objects. These are, first of all, properties of the excited atoms and molecules in gaseous state (in particular, excimer molecules, multi-charged ions and Rydberg atoms) as well as the processes involving these objects.

Chapter 6 describes the theoretical and experimental foundations of a new scientific area, which has been arisen at the interface several disciplines. We mean here the field of knowledge dealing with molecular structure and dynamics. In a broad sense, it is referred to the researches at the molecular level. An ultimate goal of such research is to elucidate electronic and space structure of multi-electronic (molecular) systems as well as the nature of the processes and phenomena occurring with their participation. In chapter 7, the quantum information, a new direction of physics originated due to the development of so far nondemanded ideas of quantum mechanics, is analyzed. In a number of publications it has been noted that the quantum information is in the beginning of its development. At the same time, this science is progressing so rapidly and its results are so impressive that they should be covered in educational literature. Chapter 8 discloses the modern ideas about the nature of fundamental forces. In chapter 9, dramatic advances in the research of nuclear structure and dynamics, attained in the second half of the twentieth century, are discussed. In this period, a special research attention has been paid to the investigations of nuclear-nuclear interactions (first of all, high-energy nuclear objects). Consequently, the deep theoretical analysis of the experimental data obtained has allowed reaching impressive success in understanding of nuclear structure and dynamics. Chapter 10 deals with some fundamental issues related to a problem of life and thinking generation. These issues are attempted to be addressed on the basis of modern physics achievements. The problems of life and thinking generation fall beyond the scope of biology and are of general scientific interest. Over the last decade, they are drawing attention of the physicists, especially those who are engaged into nonlinear tasks and self-organization problems.

The second includes five chapters. Two of them are devoted to the non-linear-optical and electrical phenomena in the materials, which are characterized by the condensed state of the matter. Optical properties of semiconducting films, the media with negative refractive index, and free electron lasers are discussed. High-temperature superconductivity, physical foundations of spintronics and acoustoelectronics, electrons in curvilinear nanostructures, collective phenomena in semiconductors involving the excitons are also described. Chapter 3 is dedicated to peculiarities of physical properties of new magnetic materials. Creation of the latter is of significant importance for the progress in science and technology, which we witnesses nowadays. In particular, physical properties of manganites and ferromagnetics with shape memory are considered. In chapter 4, the results of a new branch of knowledge, related to the space organization in the condensed media, are analyzed. Having been originated in the middle of the twentieth century owing to, first of all, works in the field of physics of open 
systems and the ideas of self-organization, this section of knowledge already plays an important role in technological progress of the society. In chapter 4, structure formation in non-linear dynamic systems, clusters and cluster beams, liquid crystal state, defect-impurity and isotopic engineering, and formation of three-dimensional nanostructures on the surface of semiconductors, are described. Finally, chapter 5 covers the main characteristics of some new analytical methods. Among a wide set of modern methods for the structural investigations, those based studies of micro and nanoobjects hold a special position. Exceptional informational value of these methods makes them predominating in a broad aspect of scientific research. Over the last fifteen - twenty years, these methods are widely employed into different branches of industry as nondestructive control methods, into medical practice as well as for environmental monitoring.

The third book is devoted to physical foundations of nanotechnologies. The book consists of three chapters. The first chapter deals with the physical phenomena and regularities related to plasma state. It should be emphasized that modern nanotechnologies require the information about plasma state of the matter obtained, mainly, for the last twenty five - thirty years. Those branches of science, which relate to the cluster formation in plasma, are especially important. In this line, the conditions of cluster plasma existence, charging of clusters and small particles in plasma, dynamics of processes in cluster plasma, methods of cluster generation are considered. Also, magnetron plasma, applications of clusters, and femtosecond excitation of the cluster beams are discussed. Special attention is paid to peculiarities of physical processes in non-ideal, dust and laser plasma.

Another field of physics, which has fundamental importance for development of nanotechnologies, relates to the condensed states at nano-scale level. We mean here the nanostructures as such, and also those incorporated in the macrosamples, first of all, at a surface of solids. The fundamental ideas, related to physics of micro and nanoworld of the solid and liquid material bodies, are discussed in the second part of the textbook. In this line, optical characteristics of the nanomaterials, physical properties of carbon nanotubes and materials on their basis, effects of dimensional quantization in the nanostructures, liquid crystals, and electroconductive polymers are considered. A separated chapter is dedicated to track- and defect formation in the condensed media.

Apart from the above chapters, the textbook also includes the materials concerning the theoretical and experimental methods for the investigations of multi-electron systems. A special attention is paid to the theoretical methods, which allow rather adequately describing, first of all, multi-particle aspects of the collective electron phenomena. The phenomenological explanation of specific properties of the metamaterials is offered. Low-dimensional effects in the nanostructures, phenomenological theories in multi-particle tasks are discussed on the example of elastic properties of quasi-crystals, cluster and phase transitions. Final chapter of the third part deals with physical foundations of new spectral research methods.

The textbook "Modern physics" can be used in such special courses as "The physics of the condensed state", "New analytical methods of research", "Physical properties of nanomaterials", and other related to, in particular, to the section "Disciplines of University Council".

\section{CONCLUSIONS}

A set of textbook under the general title "Modern Physics" is intended for senior students of the universities training engineers of industrial productions and employers of the research institutes. The textbook can also be used for students of other specialties of the natural-science 
and technical profiles in the universities, where the courses related to physical phenomena of material world at nano- and microscales, are delivered. The textbook will also be useful for teachers of the corresponding disciplines and for all who is interested in physics in its state-ofthe-art.

The three-volume edition "Modern physics" is recommended for publication by Scientific and Methodical Physical Council of the Ministry of Education and Science of the Russian Federation. Authors of this textbook are winners of the Award of the Government of the Russian Federation in the field of education (2015).

\section{References}

Orear J. Physics. M.: Mir, 1981. - V.1, 336 p.; V.2, 288 p (In Russian).

Voronov V.K., Podoplelov A.V. Modern physics. - M.: KomKniga, 2005. - 512 p.

Voronov V.K., Podoplelov A.V. Modern physics: The condensed state. - M.: LKI, 2008. - 336 p.

Voronov V.K., Podoplelov A.V., Sagdeev R.Z. Physical foundations of nanotechnologies. - M.: «LIBROKOM», 2011. $432 \mathrm{p}$. 